\title{
On the Lacunary Statistical Convergence of Triple Sequences in Fuzzy Metric Spaces
}

Mehmet Gürdal ( $\square$ gurdalmehmet@sdu.edu.tr)

Suleyman Demirel University https://orcid.org/0000-0003-0866-1869

\section{Ekrem Savaş}

Usak University: Usak Universitesi

\section{Research Article}

Keywords: Fuzzy metric space, Triple sequence, Lacunary statistically convergent sequence, Lacunary statistically Cauchy sequence, Completeness

Posted Date: April 28th, 2021

DOI: https://doi.org/10.21203/rs.3.rs-185625/v1

License: (c) (1) This work is licensed under a Creative Commons Attribution 4.0 International License. Read Full License 


\title{
ON THE LACUNARY STATISTICAL CONVERGENCE OF TRIPLE SEQUENCES IN FUZZY METRIC SPACES
}

\author{
MEHMET GÜRDAL AND EKREM SAVAŞ
}

\begin{abstract}
In this research paper, we analyze the lacunary statistical convergence and lacunary statistical Cauchy concepts of triple sequence in fuzzy metric space. We also introduce the concept of triple lacunary statistical completeness and prove some basic properties.
\end{abstract}

\section{Introduction}

In recent years, the fuzzy theory has emerged as the most active area of research in many branches of mathematics and engineering. In 1965, Zadeh [34] first introduced the fuzzy set theory and since then a large number of research papers have appeared by using the concept of fuzzy set (numbers) and fuzzification of many classical theories has also been made. One of the main problems in the theory of fuzzy topological spaces is to obtain an appropriate and consistent notion of a fuzzy metric space. Many authors have defined several concepts of fuzzy metric space in different ways $[2,8,17,18]$. In $[8,9]$, George and Veeramani introduced and studied the concept of fuzzy metric space (in short FMS) with the help of continuous $t$-norms. Recently, several convergences in fuzzy metric spaces, as $p$-convergence, $s$-convergence, st-convergence and std-convergence, were studied by Gregori et al $[12,13,14,15]$.

Freedman and Sember introduced the concept of lower asymptotic density and defined the concept of convergence in density in [5]. Fast [4] officially introduced the definition of statistical convergence. Šalát [26] gave some basic properties of statistical convergence. The study of statistical convergence in triple sequence has been initiated by Şahiner et al. [25]. Fridy and Orhan defined the concept of lacunary statistical convergence [7]. Various applications of this concept can be found in $[3,6,10,16,20,21,22,23,24,27,28,29,30,32,33]$.

In this research paper, we analyze the lacunary statistical convergence and lacunary statistical Cauchy concepts of triple sequence in fuzzy metric space. We also

2000 Mathematics Subject Classification. Primary 40A05; Secondary 54A40, 54E50.

Key words and phrases. Fuzzy metric space, Triple sequence, Lacunary statistically convergent sequence, Lacunary statistically Cauchy sequence, Completeness. 
introduce the concept of lacunary statistical completeness which would provide a more general framework to study the completeness of fuzzy metric spaces.

\section{Preliminaries}

We recall the following basic concepts from $[1,8,11,18,31]$. They will be needed in the course of the paper. By $\mathbb{N}$ and $\mathbb{R}$, we mean the set of all natural and real numbers, respectively.

Definition 1. A binary operation $*:[0,1] \times[0,1] \rightarrow[0,1]$ is a continuous $t$-norm if $([0,1], *)$ is a topological monoid with unit 1 such that $a * b \leq c * d$ whenever $a \leq c$ and $b \leq d(a, b, c, d \in[0,1])$.

Example 1. $a * b=\min \{a, b\}$ and $a * b=a . b$ are two common examples of continuous t-norms.

Definition 2. The 3-tuple $(X, M, *)$ is said to be a fuzzy metric space if $X$ is a nonempty set, $*$ is a continuous $t$-norm and $M$ is a fuzzy set on $X^{2} \times(0, \infty)$ satisfying the following conditions for all $x, y, z \in X$ and $s, t>0$ :

(i) $M(x, y, t)>0$;

(ii) $M(x, y, t)=1$ if and only if $x=y$;

(iii) $M(x, y, t)=M(y, x, t)$;

(iv) $M(x, y, t) * M(y, z, s) \leq M(x, z, t+s)$;

(v) $M(x, y,):.(0, \infty) \rightarrow[0,1]$ is continuous.

If $(X, M, *)$ is a fuzzy metric space, then we will call $(M, *)$, or simply $M$, a fuzzy metric on $X$.

Let $(X, d)$ be a metric space. Denote by $a . b$ the usual multiplication for all $a, b \in[0,1]$, and let $M_{d}$ be the fuzzy set defined on $X^{2} \times(0, \infty)$ by

$$
M_{d}(x, y, t)=\frac{t}{t+d(x, y)} .
$$

Then $\left(M_{d},.\right)$ is a fuzzy metric on $X$ called standard fuzzy metric space [8].

Definition 3. Let $(X, M, *)$ be a fuzzy metric space. We define open ball $B_{M}(x, r, t)$ with centre $x \in X$ and radius $r, 0<r<1, t>0$ as

$$
B_{M}(x, r, t)=\{y \in X: M(x, y, t)>1-r\} .
$$

Let $(X, M, *)$ be a fuzzy metric space. Define $\tau_{M}=\left\{A \subset X: x \in A\right.$ iff there exists $t>0, r \in(0,1)$ such that $\left.B_{M}(x, r, t) \subset A\right\}$. Then $\tau_{M}$ is a topology on $X$. George and Veeramani [8] proved that $\left\{B_{M}(x, r, t)\right.$ : $x \in X, t>0, r \in(0,1)\}$ forms a base of a topology $\tau_{M}$ in $X$. 
The next characterization of convergent sequences was gave in $[8]$.

Proposition 1. Let $(X, M, *)$ be a fuzzy metric space and $\tau_{M}$ be the topology induced by the fuzzy metric. Then for a sequence $\left\{x_{n}\right\}$ in $X, x_{n}$ converges to $x$ in $X$ if and only if $M\left(x_{n}, x, t\right)$ tends to 1 as $n$ tends to $\infty$, for $t>0$.

It is easy to see that $\left\{x_{n}\right\}$ converges to $x_{0}$ which is equivalent to the following :

For each $r \in(0,1)$ and each $t>0$, there exists $n_{0} \in \mathbb{N}$ such that $M\left(x_{n}, x_{0}, t\right)>$ $1-r$ for all $n>n_{0}$.

Definition 4. A sequence $\left\{x_{n}\right\}$ in a fuzzy metric space $(X, M, *)$ is said to be a Cauchy sequence if for each $\varepsilon, 0<\varepsilon<1$ and $t>0$, there exists $n_{0} \in \mathbb{N}$ such that $M\left(x_{n}, x_{m}, t\right)>1-\varepsilon$ for all $n, m \geq n_{0}$.

Definition 5. A fuzzy metric space is said to be complete if every Cauchy sequence is convergent.

\section{Triple Lacunary statistical CONVERGence in FMS}

Li et al. [19] introduced the concepts of statistical convergence in fuzzy metric spaces. In this section, we present the notion of lacunary statistically convergent and lacunary statistically Cauchy of triple sequences in fuzzy metric spaces.

The idea is based on the notion of natural density of subsets of $\mathbb{N}$, the set of all positive integers which is defined as follows: The natural density of a subset $A$ of $\mathbb{N}$ denoted as $\delta(A)$ is defined by $\delta(A)=\lim _{n \rightarrow \infty} \frac{1}{n}|\{k \leq n: k \in A\}|$. Here, for a subset $K$ of a set $X,|K|$ will denote the cardinality of $K$. It is straightforward to show that $\delta(A) \in[0,1]$ and $\delta(\mathbb{N}-A)=1-\delta(A)$ if $\delta(A)$ exists. $A$ is said to be statistically dense provided that $\delta(A)=1$.

We now recall that the concept of statistical convergence for triple sequences was presented by Şahiner, Gürdal and Düden [25] as follows:

A function $x: \mathbb{N} \times \mathbb{N} \times \mathbb{N} \rightarrow \mathbb{R}$ (or $\mathbb{C}$ ) is called a real (complex) triple sequence. A triple sequence $\left(x_{j k l}\right)$ is said to be convergent to $L$ in Pringsheim's sense if for every $\varepsilon>0$, there exists $n_{0}(\varepsilon) \in N$ such that $\left|x_{j k l}-L\right|<\varepsilon$ whenever $j, k, l \geq n_{0}$.

Definition 6. A subset $K$ of $\mathbb{N} \times \mathbb{N} \times \mathbb{N}$ is said to have natural density $\delta_{3}(K)$ if

$$
\delta_{3}(K)=P-\lim _{n, k, l \rightarrow \infty} \frac{\left|K_{n k l}\right|}{n k l}
$$

exists, where the vertical bars denote the number of $(n, k, l)$ in $K$ such that $p \leq n$, $q \leq k, r \leq l$. Then, a real triple sequence $x=\left(x_{p q r}\right)$ is said to be statistically convergent to $L$ in Pringsheim's sense if for every $\varepsilon>0$,

$$
\delta_{3}\left(\left\{(n, k, l) \in \mathbb{N} \times \mathbb{N} \times \mathbb{N}: p \leq n, q \leq k, r \leq l,\left|x_{p q r}-L\right| \geq \varepsilon\right\}\right)=0 .
$$


Furthermore, a new type of sequence called triple lacunary sequence was introduced in Esi and Savaş [3]. The triple sequence $\theta_{3}=\theta_{r, s, t}=\left\{\left(j_{r}, k_{s}, l_{t}\right)\right\}$ is called triple lacunary sequence if there exist three increasing sequences of integers such that

$$
\begin{gathered}
j_{0}=0, h_{r}=j_{r}-j_{r-1} \rightarrow \infty \text { as } r \rightarrow \infty, \\
k_{0}=0, h_{s}=k_{s}-k_{s-1} \rightarrow \infty \text { as } s \rightarrow \infty,
\end{gathered}
$$

and

$$
l_{0}=0, h_{t}=l_{t}-l_{t-1} \rightarrow \infty \text { as } t \rightarrow \infty .
$$

Let $k_{r, s, t}=j_{r} k_{s} l_{t}, h_{r, s, t}=h_{r} h_{s} h_{t}$ and $\theta_{r, s, t}$ is determined by

$$
\begin{gathered}
I_{r, s, t}=\left\{(j, k, l): j_{r-1}<j \leq j_{r}, k_{s-1}<k \leq k_{s} \text { and } l_{t-1}<l \leq l_{t}\right\}, \\
q_{r}=\frac{j_{r}}{j_{r-1}}, q_{s}=\frac{k_{s}}{k_{s-1}}, q_{t}=\frac{l_{t}}{l_{t-1}} \text { and } q_{r, s, t}=q_{r} q_{s} q_{t} .
\end{gathered}
$$

Let $D \subset \mathbb{N} \times \mathbb{N} \times \mathbb{N}$. The number

$$
\delta_{\theta_{3}}(D)=\lim _{r, s, t} \frac{1}{h_{r, s, t}}\left|\left\{(j, k, l) \in I_{r, s, t}:(j, k, l) \in D\right\}\right|
$$

is said to be the $\theta_{r, s, t}$-density of $D$, provided the limit exists.

We start this section with the following definition.

Definition 7. Let $(X, M, *)$ be a fuzzy metric space. A triple sequence $\left\{x_{j k l}\right\}$ in $X$ is said to be convergent to $L \in X$ if, for each $r \in(0,1)$ and each $t>0$, there exists $n_{0} \in \mathbb{N}$ such that

$$
M\left(x_{j k l}, L, t\right)>1-r \text { whenever } j>n_{0}, k>n_{0}, l>n_{0} .
$$

Definition 8. Let $(X, M, *)$ be a fuzzy metric space. A triple sequence $\left\{x_{j k l}\right\}$ in $X$ is said to be statistically convergent to $L \in X$ if

$$
\delta\left(\left\{(j, k, l) \in \mathbb{N} \times \mathbb{N} \times \mathbb{N}: M\left(x_{j k l}, L, t\right)>1-r\right\}\right)=1
$$

for all $r \in(0,1)$ and $t>0$.

In this case we write $x_{j k l} \rightarrow L\left(S\left(\operatorname{sts}_{3}\right)\right)$. In such a case we say that $\left\{x_{j k l}\right\}$ is sts $_{3}$-convergent to $L$ (or, $\left\{x_{j k l}\right\} \mathrm{sts}_{3}$-converges to $L$ ). It is obvious that

$$
\begin{aligned}
\delta\left(\left\{(j, k, l) \in \mathbb{N} \times \mathbb{N} \times \mathbb{N}: M\left(x_{j k l}, L, t\right)>1-r\right\}\right) & =1 \Leftrightarrow \\
\lim _{r, s, t \rightarrow \infty} \frac{\left|\left\{j \leq r, k \leq s, l \leq t: M\left(x_{j k l}, L, \varepsilon\right)>1-r\right\}\right|}{r s t} & =1 .
\end{aligned}
$$


Example 2. Let $X=[0,3]$. Denote $a * b=a b$ and for all $a, b \in[0,1]$. For all $x, y \in \mathbb{R}$ and every $t>0$, consider

$$
M(x, y, t)=\frac{t}{t+|x-y|} .
$$

In this case observe that $(X, M, *)$ is a fuzzy metric space. Now define a sequence $x=\left\{x_{j k l}\right\}$ whose terms are given by

$$
x_{j k l}=\left\{\begin{array}{cc}
j k l, & j, k, l=\frac{r(r+3)}{2}, r \in \mathbb{N} \\
0, & \text { otherwise. }
\end{array}\right.
$$

It is immediate to see that $\left\{x_{j k l}\right\}$ is not convergent to 0, but $\left\{x_{j k l}\right\}$ is $\operatorname{sts}_{3}$-convergent to 0 .

Definition 9. Let $(X, M, *)$ be a fuzzy metric space and $\theta_{3}=\theta_{r, s, t}$ be a lacunary triple sequence. A triple sequence $\left\{x_{j k l}\right\}$ is said to be lacunary statistically convergent to $L \in X$, written as $\operatorname{sts}_{\theta_{3}}-\lim x_{j k l}=L$, if, for all $r \in(0,1)$ and $t>0$,

$$
\lim _{r, s, t} \frac{1}{h_{r, s, t}}\left|\left\{(j, k, l) \in I_{r, s, t}: M\left(x_{j k l}, L, t\right)>1-r\right\}\right|=1
$$

or

$$
\delta_{\theta}\left(\left\{(j, k, l) \in \mathbb{N} \times \mathbb{N} \times \mathbb{N}: M\left(x_{j k l}, L, t\right)>1-r\right\}\right)=1 .
$$

In this case we write $x_{j k l} \rightarrow L\left(S_{\theta_{3}}(\mathrm{sts})\right)$, where $L$ is said to be $\operatorname{sts}_{\theta_{3}}-\lim$.

Theorem 1. Let $(X, M, *)$ be a fuzzy metric space and $\theta_{3}=\theta_{r, s, t}$ be a lacunary triple sequence. If a triple sequence $\left\{x_{j k l}\right\}$ in $X$ is lacunary statistically convergent in $X$, then $\operatorname{sts}_{\theta_{3}}-\lim$ is unique.

Proof. Suppose that $x_{j k l} \rightarrow L_{1}\left(S_{\theta_{3}}\right.$ (sts)) and $x_{j k l} \rightarrow L_{2}\left(S_{\theta_{3}}\right.$ (sts)). Let $L_{1}$ and $L_{2}$ be two distinct points in $X$ and $t>0$. Then $0<M\left(L_{1}, L_{2}, t\right)<1$. Put $1-\varepsilon \in\left(M\left(L_{1}, L_{2}, t\right), 1\right)$. For each $1-s \in(1-\varepsilon, 1)$, there exists $1-s$ such that $(1-s) *(1-s) \geq 1-\varepsilon$. Let

$$
B_{L_{1}}=\left\{y \in X: M\left(L_{1}, y, \frac{t}{2}\right)>1-s\right\}
$$

and

$$
B_{L_{2}}=\left\{y \in X: M\left(L_{2}, y, \frac{t}{2}\right)>1-s\right\} .
$$

We claim that $B_{L_{1}} \cap B_{L_{2}}=\emptyset$. In fact, if there exists $z \in B_{L_{1}} \cap B_{L_{2}}$, then we have

$$
\begin{aligned}
M\left(L_{1}, L_{2}, t\right) & \geq M\left(L_{1}, z, \frac{t}{2}\right) * M\left(z, L_{2}, \frac{t}{2}\right) \\
& \geq(1-s) *(1-s) \geq 1-\varepsilon \\
& >M\left(L_{1}, L_{2}, t\right)
\end{aligned}
$$


which is a contradiction. Since

$$
\left\{y \in X: M\left(L_{2}, y, \frac{t}{2}\right)>1-s\right\} \subset\left\{x \in X: M\left(x, L_{1}, \frac{t}{2}\right) \leq 1-s\right\},
$$

it follows that

$$
\begin{aligned}
& \left\{(j, k, l) \in \mathbb{N} \times \mathbb{N} \times \mathbb{N}: M\left(x_{j k l}, L_{2}, \frac{t}{2}\right)>1-s\right\} \\
& \subseteq\left\{(j, k, l) \in \mathbb{N} \times \mathbb{N} \times \mathbb{N}: M\left(x_{j k l}, L_{1}, \frac{t}{2}\right) \leq 1-s\right\} .
\end{aligned}
$$

Then we may write

$$
\begin{aligned}
& \lim _{r, s, t} \frac{1}{h_{r, s, t}}\left|\left\{(j, k, l) \in I_{r, s, t}: M\left(x_{j k l}, L_{1}, \frac{t}{2}\right) \leq 1-s\right\}\right| \\
& =1-\lim _{r, s, t} \frac{1}{h_{r, s, t}}\left|\left\{(j, k, l) \in I_{r, s, t}: M\left(x_{j k l}, L_{1}, \frac{t}{2}\right)>1-s\right\}\right| \\
& =1-1=0 .
\end{aligned}
$$

By (1), we get

$$
\begin{aligned}
1 & =\lim _{r, s, t} \frac{1}{h_{r, s, t}}\left|\left\{(j, k, l) \in I_{r, s, t}: M\left(x_{j k l}, L_{2}, \frac{t}{2}\right)>1-s\right\}\right| \\
& \leq \lim _{r, s, t} \frac{1}{h_{r, s, t}}\left|\left\{(j, k, l) \in I_{r, s, t}: M\left(x_{j k l}, L_{1}, \frac{t}{2}\right) \leq 1-s\right\}\right|=0 .
\end{aligned}
$$

which is a contradiction. Therefore, we conclude that $s t s_{\theta_{3}}$-lim is unique. This completes the proof of the theorem.

Theorem 2. Let $(X, M, *)$ be a fuzzy metric space and $\theta_{3}=\theta_{r, s, t}$ be a lacunary triple sequence. If triple sequence $\left\{x_{j k l}\right\}$ in $X$ is convergent to $L$, then $\left\{x_{j k l}\right\}$ is lacunary statistical convergent to L. But converse need not be true.

Proof. Suppose that $\left\{x_{j k l}\right\}$ is convergent to $L$. Let $r \in(0,1)$ and $t>0$. Then there exists $n_{0} \in \mathbb{N}$ such that $M\left(x_{j k l}, L, t\right)>1-r$ for all $j>n_{0}, k>n_{0}, l>n_{0}$. Hence

$$
\left|\left\{(j, k, l) \in \mathbb{N} \times \mathbb{N} \times \mathbb{N}: M\left(x_{j k l}, L, \varepsilon\right)>1-r\right\}\right| \geq\left(j-j_{0}\right)\left(k-k_{0}\right)\left(l-l_{0}\right) .
$$

Therefore

$$
\lim _{r, s, t} \frac{1}{h_{r, s, t}}\left|\left\{(j, k, l) \in I_{r, s, t}: M\left(x_{j k l}, L, t\right)>1-r\right\}\right|=1 .
$$

that is $x_{j k l} \rightarrow L\left(S_{\theta_{3}}(\mathrm{sts})\right)$. We complete the proof.

The following example shows that the converse of Theorem 2 is not valid.

Example 3. Let $(\mathbb{R},||$.$) denote the space of real numbers with the usual metric,$ and let $a * b=a b$ and for all $a, b \in[0,1]$. For all $x, y \in \mathbb{R}$ and every $t>0$, consider

$$
M(x, y, t)=\frac{t}{t+|x-y|}
$$


In this case observe that $(\mathbb{R}, M, *)$ is a fuzzy metric space (see [8]). Now define a sequence $x=\left\{x_{j k l}\right\}$ whose terms are given by

$$
x_{j k l}=\left\{\begin{array}{cc}
j_{r}-\left[\sqrt{h_{r}}\right]+1 \leq j \leq j_{r}, \\
j k l, & k_{s}-\left[\sqrt{h_{s}}\right]+1 \leq s \leq k_{s}, \\
& l_{t}-\left[\sqrt{h_{t}}\right]+1 \leq l \leq l_{t}, r, s, t \in \mathbb{N} \\
0, & \text { otherwise. }
\end{array}\right.
$$

It is immediate to see that $\left\{x_{j k l}\right\}$ is not convergent to 0 . We claim that $\left\{x_{j k l}\right\}$ is $\operatorname{sts}_{\theta_{3}}$-convergent to 0 . In fact, let $r \in(0,1)$ and $t>0$. Put

$$
A=\left\{(j, k, l) \in \mathbb{N} \times \mathbb{N} \times \mathbb{N}: M\left(x_{j k l}, x, t\right) \leq 1-r\right\} .
$$

Then

$$
\begin{aligned}
A & =\left\{(j, k, l) \in \mathbb{N} \times \mathbb{N} \times \mathbb{N}: M\left(x_{j k l}, x, t\right) \leq 1-r\right\} \\
& =\left\{(j, k, l) \in \mathbb{N} \times \mathbb{N} \times \mathbb{N}: j_{r}-\left[\sqrt{h_{r}}\right]+1 \leq j \leq j_{r},\right. \\
& \left.k_{s}-\left[\sqrt{h_{s}}\right]+1 \leq k \leq k_{s}, l_{t}-\left[\sqrt{h_{t}}\right]+1 \leq l \leq l_{t}, r, s, t \in \mathbb{N}\right\} \\
& =K
\end{aligned}
$$

and so, we get

which implies that

$$
\frac{1}{h_{r, s, t}}|A| \leq \frac{1}{h_{r, s, t}}|K| \leq \frac{\sqrt{h_{r}} \sqrt{h_{s}} \sqrt{h_{t}}}{h_{r, s, t}}
$$

$$
\lim \frac{1}{h_{r, s, t}}\left|\left\{(j, k, l) \in I_{r, s, t}: M\left(x_{j k l}, x, t\right) \leq 1-r\right\}\right|=0
$$

Consequently,

$$
\lim \frac{1}{h_{r, s, t}}\left|\left\{(j, k, l) \in I_{r, s, t}: M\left(x_{j k l}, 0, t\right)>1-r\right\}\right|=1
$$

for all $r \in(0,1)$ and $t>0$.

Theorem 3. Let $x=\left\{x_{j k l}\right\}$ be a triple sequence in a fuzzy metric space $(X, M, *)$. Then, for any lacunary triple sequence $\theta_{3}, \operatorname{sts}_{\theta_{3}}-\lim x=L$ if and only if there exists a subset $K=\{(j, k, l): j, k, l=1,2, \ldots\} \subset \mathbb{N} \times \mathbb{N} \times \mathbb{N}$ such that $\delta_{\theta_{3}}(K)=1$ and $\left\{x_{j k l}\right\}_{(j, k, l) \in K}$ converges to $L$.

Proof. Suppose that $\operatorname{sts}_{\theta_{3}}-\lim x=L$. Let

$$
K_{s}=\left\{(j, k, l) \in \mathbb{N} \times \mathbb{N} \times \mathbb{N}: M\left(x_{j k l}, L, t\right)>1-\frac{1}{s}\right\} .
$$

Then, for $t>0$ and each $s=1,2, \ldots$, we have that

$$
\delta_{\theta_{3}}\left(K_{s}\right)=1
$$


and

$$
K_{s+1} \subseteq K_{s} .
$$

For $(j, k, l) \in K_{s}$, we need to show that $\left\{x_{j k l}\right\}$ converges to $L$. Now, suppose that $\left\{x_{j k l}\right\}$ is not convergent to $L$. Therefore there is $\mu>0$ such that

$$
\left\{(j, k, l) \in \mathbb{N} \times \mathbb{N} \times \mathbb{N}: M\left(x_{j k l}, L, t\right) \leq 1-\mu\right\}
$$

for inifinitely many terms. Let

$$
K_{\mu}=\left\{(j, k, l) \in \mathbb{N} \times \mathbb{N} \times \mathbb{N}: M\left(x_{j k l}, L, t\right) \leq 1-\mu\right\}, \mu>\frac{1}{s} .
$$

Then $\delta_{\theta_{3}}\left(K_{\mu}\right)=0$ and by $(2), K_{s} \subset K_{\mu}$. Hence $\delta_{\theta_{3}}\left(K_{s}\right)=0$ which contradics (3). So $\left\{x_{j k l}\right\}$ converges to $L$.

Conversely, suppose that there exists a subset $K=\{(j, k, l): j, k, l=1,2, \ldots\} \subset$ $\mathbb{N} \times \mathbb{N} \times \mathbb{N}$ such that $\delta_{\theta_{3}}(K)=1$ and $\left\{x_{j k l}\right\}$ converges to $L$, i.e. there exists $k_{0} \in \mathbb{N}$ such that all $r \in(0,1)$ and $t>0$,

$$
M\left(x_{j k l}, L, t\right)>1-r, \forall j, k, l \geq k_{0}
$$

Now

$$
\begin{aligned}
K_{M}(r, t) & =\left\{(j, k, l) \in \mathbb{N} \times \mathbb{N} \times \mathbb{N}: M\left(x_{j k l}, L, t\right) \leq 1-r\right\} \\
& \subseteq \mathbb{N} \times \mathbb{N} \times \mathbb{N}-\left\{\left(j_{k_{0}+1}, k_{k_{0}+1}, l_{k_{0}+1}\right),\left(j_{k_{0}+2}, k_{k_{0}+2}, l_{k_{0}+2}\right), \ldots\right\} .
\end{aligned}
$$

This implies that $\delta_{\theta_{3}}\left(K_{M}(r, t)\right) \leq 1-1=0$. Therefore

$$
\lim _{r, s, t} \frac{1}{h_{r, s, t}}\left|\left\{(j, k, l) \in I_{r, s, t}: M\left(x_{j k l}, L, t\right)>1-r\right\}\right|=1 .
$$

Hence $\operatorname{sts}_{\theta_{3}}-\lim x_{j k l}=L$. This completes the proof of the theorem.

\section{TRIPle LaCUnARY STATistically COMPlete FUZZY METRIC SPACES}

In [19], Li et al. has defined the concept of statistically Cauchy sequences in fuzzy metric space. In this section we define lacunary statistically Cauchy triple sequences with respect to an fuzzy metric space. And we consider some relations between lacunary statistical completeness and the ordinary completeness in fuzzy metric space. Now, we define the Cauchy and lacunary statistical convergence of triple sequences in a fuzzy metric space.

Definition 10. Let $(X, M, *)$ be a fuzzy metric space. A triple sequence $\left\{x_{j k l}\right\}$ in $X$ is called Cauchy if for each $\alpha \in(0,1)$ and each $t>0$, there exists $N \in \mathbb{N}$ such that

$$
M\left(x_{j k l}, x_{p q r}, t\right)>1-\alpha
$$

whenever $j \geq p \geq N, k \geq q \geq N, l \geq r \geq N$. 
Definition 11. Let $(X, M, *)$ be a fuzzy metric space and $\theta_{3}=\theta_{r, s, t}$ be a lacunary triple sequence. A triple sequence $\left\{x_{j k l}\right\}$ in $X$ is said to be lacunary statistically Cauchy sequence, if, for all $\alpha \in(0,1)$ and $t>0$, there exists $M, M \prime, M^{\prime \prime} \in \mathbb{N}$ such that for all $j, p \geq M^{\prime \prime}, k, q \geq M^{\prime}, l, r \geq M$,

$$
\delta_{\theta_{3}}\left(\left\{(j, k, l) \in \mathbb{N} \times \mathbb{N} \times \mathbb{N}: M\left(x_{j k l}, x_{p q r}, t\right)>1-\alpha\right\}\right)=1 .
$$

In such a case we say that $\left\{x_{j k l}\right\}$ is $\operatorname{sts}_{\theta_{3}}$-Cauchy.

Theorem 4. Let $\left\{x_{j k l}\right\}$ be a triple sequence in a fuzzy metric space $(X, M, *)$ and $\theta_{3}=\theta_{r, s, t}$ be a lacunary triple sequence. If $\left\{x_{j k l}\right\}$ is a lacunary statistically convergent, then $\left\{x_{j k l}\right\}$ is a lacunary statistically Cauchy.

Proof. Let $\left\{x_{j k l}\right\}$ be lacunary statistically convergent to $L$, i.e., $x_{j k l} \rightarrow L\left(S_{\theta_{3}}\right.$ (sts)). Let $\alpha \in(0,1)$ and $t>0$. Then there exists $\alpha_{1} \in(0, \alpha)$ such that $\left(1-\alpha_{1}\right) *\left(1-\alpha_{1}\right)>$ $1-\alpha$. According to Theorem 3, there exists a subset $K=\{(j, k, l): j, k, l=$ $1,2, \ldots\} \subset \mathbb{N} \times \mathbb{N} \times \mathbb{N}$ such that $\delta_{\theta_{3}}(K)=1$ and $\left\{x_{j k l}\right\}$ converges to $L$. Thus there exists $n_{0} \in \mathbb{N}$ such that

$$
M\left(x_{j k l}, L, \frac{t}{2}\right)>1-\alpha_{1} \text { for all } j>n_{0}, k>n_{0}, l>n_{0} .
$$

Let $(p, q, r) \in\left\{(j, k, l) \in \mathbb{N} \times \mathbb{N} \times \mathbb{N}: M\left(x_{j k l}, L, \frac{t}{2}\right)>1-\alpha_{1}\right\}$. Then

$$
\begin{aligned}
M\left(x_{p q r}, x_{j k l}, t\right) & \geq M\left(x_{p q r}, L, t / 2\right) * M\left(x_{j k l}, L, t / 2\right) \\
& \geq\left(1-\alpha_{1}\right) *\left(1-\alpha_{1}\right)>1-\alpha .
\end{aligned}
$$

It follows that

$$
\begin{aligned}
& \left\{(j, k, l) \in \mathbb{N} \times \mathbb{N} \times \mathbb{N}: M\left(x_{j k l}, L, \frac{t}{2}\right)>1-\alpha_{1}\right\} \\
& \subseteq\left\{(j, k, l) \in \mathbb{N} \times \mathbb{N} \times \mathbb{N}: M\left(x_{j k l}, x_{p q r}, t\right)>1-\alpha_{1}\right\} .
\end{aligned}
$$

Since $\delta_{\theta_{3}}\left(\left\{(j, k, l) \in \mathbb{N} \times \mathbb{N} \times \mathbb{N}: M\left(x_{j k l}, L, \frac{t}{2}\right)>1-\alpha_{1}\right\}\right)=1$ and (4), we get that

$$
\delta_{\theta_{3}}\left(\left\{(j, k, l) \in \mathbb{N} \times \mathbb{N} \times \mathbb{N}: M\left(x_{j k l}, x_{p q r}, t\right)>1-\alpha_{1}\right\}\right)=1 .
$$

So $\left\{x_{j k l}\right\}$ is $\operatorname{sts}_{\theta_{3}}$-Cauchy. This completes the proof of the theorem.

The following example shows that the converse of the above theorem need not be true.

Example 4. Let $X=\left\{x_{j k l}:(j, k, l) \in \mathbb{N} \times \mathbb{N} \times \mathbb{N}\right\}$, where $x_{j k l}=1-\frac{1}{(j+1)(k+1)(l+1)}$ $(j, k, l \in \mathbb{N})$ and $a * b=\min \{a, b\}$ for all $a, b \in[0,1]$, and let $M$ be a fuzzy set on $X^{2} \times(0, \infty)$ defined as follows:

$$
M(x, y, t)=\left\{\begin{array}{cc}
1, & x=y \\
\min \{x, y\}, & x \neq y
\end{array}\right.
$$


for all $x, y \in X$ and $t>0$. Then $(X, M, *)$ is a fuzzy metric space and triple sequence $\left\{x_{j k l}\right\}$ in $(X, M, *)$ is sts $s_{\theta_{3}}$-Cauchy, but it is not sts $s_{\theta_{3}}$-convergent.

Let $\alpha \in(0,1)$ and $t>0$. Then there exists $p, q, r \in \mathbb{N}$ such that $\frac{1}{(p+1)(q+1)(r+1)}<$ $\alpha$. Hence

$$
M\left(x_{j k l}, x_{p q r}, t\right)=x_{p q r}=1-\frac{1}{(p+1)(q+1)(r+1)}>1-\alpha
$$

for all $j>p, k>q, l>r$. Thus $\delta_{\theta}\left(\left\{(j, k, l) \in \mathbb{N} \times \mathbb{N} \times \mathbb{N}: M\left(x_{j k l}, x_{p q r}, t\right)>\right.\right.$ $1-\alpha\})=1$ which means that $\left\{x_{j k l}\right\}$ is sts $s_{\theta_{3}}$-Cauchy. Let $L \in X$. Then there exists $p, q, r \in \mathbb{N}$ such that $L=x_{p q r}=1-\frac{1}{(p+1)(q+1)(r+1)}$. Now, fix $t_{0}=\alpha_{0}=$ $\frac{1}{3(p+1)(q+1)(r+1)}$. Then

$$
M\left(x_{j k l}, L, t_{0}\right)=M\left(x_{j k l}, x_{p q r}, t_{0}\right)=x_{p q r}=1-\frac{1}{(p+1)(q+1)(r+1)} \leq 1-\alpha_{0}
$$

for all $j>p, k>q, l>r$. Hence

$$
\delta_{\theta}\left(\left\{(j, k, l) \in \mathbb{N} \times \mathbb{N} \times \mathbb{N}: M\left(x_{j k l}, L, t_{0}\right) \leq 1-\alpha_{0}\right\}\right)=1,
$$

which implies that $\delta_{\theta}\left(\left\{(j, k, l) \in \mathbb{N} \times \mathbb{N} \times \mathbb{N}: M\left(x_{j k l}, L, t_{0}\right)>1-\alpha_{0}\right\}\right)=0$. So $\left\{x_{j k l}\right\}$ is not sts $s_{\theta_{3}}$-convergent.

Corollary 1. If a triple sequence in a fuzzy metric space is Cauchy, then it is $\operatorname{sts}_{\theta_{3}}$-Cauchy. However, the converse is false.

Now using a similar technique in the proof of Theorem 3, one can get the following result at once.

Theorem 5. Let $(X, M, *)$ be a fuzzy metric space, and let $x=\left\{x_{j k l}\right\}$ be a triple sequence whose terms are in $X$. Then, for $\theta_{3}=\theta_{r, s, t}$ lacunary triple sequence, the following conditions are equivalent:

(i) $x$ is $\operatorname{sts}_{\theta_{3}}$-Cauchy.

(ii) there exists an increasing index sequence $K=\{(j, k, l): j, k, l=1,2, \ldots\} \subset$ $\mathbb{N} \times \mathbb{N} \times \mathbb{N}$ such that $\delta_{\theta_{3}}(K)=1$ and the subsequence $\left\{x_{j k l}\right\}_{(j, k, l) \in K}$ is a Cauchy sequence with respect to fuzzy metric $M$.

Now, we introduce the lacunary statistically complete fuzzy metric space using the lacunary statistical Cauchy triple sequence defined above.

Definition 12. The fuzzy metric space $(X, M, *)$ is called triple lacunary statistically complete if every lacunary statistically Cauchy triple sequence in $X$ lacunary statistically converges to a point in X.

In such a case we say that $(X, M, *)$ is $\operatorname{sts}_{\theta_{3}}$-complete. 
Theorem 6. Let $(X, M, *)$ be a fuzzy metric space. If $X$ is $\operatorname{sts}_{\theta_{3}}$-complete, then it is complete.

Proof. Let $\left\{x_{j k l}\right\}$ be a Cauchy sequence in $X$. Let $\alpha \in(0,1)$ and $t>0$. Then there exists $\alpha_{1} \in(0, \alpha)$ such that $\left(1-\alpha_{1}\right) *\left(1-\alpha_{1}\right)>1-\alpha$. Therefore there exists $N \in \mathbb{N}$ such that

$$
M\left(x_{j k l}, x_{p q r}, t\right)>1-\alpha_{1}
$$

whenever $j \geq p \geq N, k \geq q \geq N, l \geq r \geq N$. Then $\left\{x_{j k l}\right\}$ is $\operatorname{sts}_{\theta_{3}}$-Cauchy by the above Corollary 1 . Since $X$ is $\operatorname{sts}_{\theta_{3}}$-complete, there exists an $L \in X$ such that $\left\{x_{j k l}\right\}$ lacunary statistically converges to $L$. Now, we show that $\left\{x_{j k l}\right\}$ converges to this $L$ in $X$. Thus, by Theorem 3, there exists an increasing index sequence $K=\{(j, k, l): j, k, l=1,2, \ldots\} \subset \mathbb{N} \times \mathbb{N} \times \mathbb{N}$ such that $\delta_{\theta_{3}}(K)=1$ and the subsequence $\left\{x_{j k l}\right\}_{(j, k, l) \in K}$ converges to $L$. Then there exists $(p, q, r) \in K$ with $n_{0} \geq N$ such that

$$
M\left(x_{p q r}, L, \frac{t}{2}\right)>1-\alpha_{1} \text { for all } p>n_{0}, q>n_{0}, r>n_{0} .
$$

Hence

$$
\begin{aligned}
M\left(x_{j k l}, L, t\right) & \geq M\left(x_{j k l}, x_{p q r}, \frac{t}{2}\right) * M\left(x_{p q r}, L, \frac{t}{2}\right) \\
& \geq\left(1-\alpha_{1}\right) *\left(1-\alpha_{1}\right)>1-\alpha
\end{aligned}
$$

for all $j \geq n_{0} \geq N, k \geq n_{0} \geq N, l \geq n_{0} \geq N$. That is $\left\{x_{j k l}\right\}$ converges to $L$, therefore $X$ is complete. This completes the proof.

\section{CONCLUding REMARKS}

In this paper, we have dealt with the notion of the lacunary statistical convergence and lacunary statistical Cauchy of triple sequence in fuzzy metric space. However, further investigation in these aspects is required. We also introduce the concept of triple lacunary statistical completeness and prove some of the basic properties.

\section{Compliance with ethical standards}

Conflict of interest The authors declare that they have no conflict of interest.

Ethical approval This article does not contain any studies with human participants \& animals performed by any of the authors.

Informed Consent Informed consent was obtained from all individual participants included in the study. 
The Authorship contributions Conceptualization: Mehmet Gürdal, Ekrem Savaş; Investigation: Mehmet Gürdal, Ekrem Savaş; Methodology: Mehmet Gürdal, Ekrem Savaş; Writing -original draft: Mehmet Gürdal, Ekrem Savaş; Writing - review \& editing: Mehmet Gürdal, Ekrem Savaş

\section{REFERENCES}

[1] R. Engelking, General Topology, PWN-Polish Science Publishers, warsaw, 1977.

[2] M.A. Erceg, Metric spaces in fuzzy set theory, J. Math. Anal. Appl., 69 (1979), 205-230.

[3] A. Esi, E. Savaş, On lacunary statistically convergent triple sequences in probabilistic normed space, Appl. Math. Inf. Sci., 9(5) (2015), 2529-2534.

[4] H. Fast, Sur la convergence statistique, Colloq. Math., 2 (1951), 241-244.

[5] A.R. Freedam, J.J. Sember, Densities and summability, Pac. J. Math., 95(2) (1981), 293-305.

[6] J.A. Fridy, On statistical convergence, Analysis (Munich), 5 (1985), 301-313.

[7] J.A. Fridy, C. Orhan, Lacunary statistical convergence, Pacific J. Math., 160 (1993), 43-51.

[8] A. George, P. Veeramani, On some results in fuzzy metric spaces, Fuzzy Sets and Systems, 64 (1994), 395-399.

[9] A. George, P. Veeramani, On some results of analysis for fuzzy metric spaces, Fuzzy Sets and Systems, 90 (1997), 365-368.

[10] M. Gürdal, S. Pehlivan, Statistical convergence in 2-normed spaces, Southeast Asian Bull. Math., 33(2) (2009), 257-264.

[11] M. Grabiec, Fixed points in fuzzy metric spaces, Fuzzy Sets and Systems 27 (1988), 385-389.

[12] V. Gregori, A. López-Crevillén, S. Morillas, A. Sapena, On convergence in fuzzy metric spaces, Topology Appl., 156 (2009), 3002-3006.

[13] V. Gregori, J. J. Miñana, std-convergence in fuzzy metric spaces, Fuzzy Sets and Systems, 267 (2015), 140-143.

[14] V. Gregori, J. J. Miñana, S. Morillas, A note on convergence in fuzzy metric spaces, Topology Appl., 163 (2014), 142-148.

[15] V. Gregori, J. J. Miñana, S. Morillas, A. Sapena, Cauchyness and convergence in fuzzy metric spaces, RACSAM Rev. R. Acad. Cienc. Exactas Fis. Nat. Ser. A Mat. ,111(1) (2017), 25-37.

[16] B. Hazarika, A. Alotaibi, S. A. Mohiudine, Statistical convergence in measure for double sequences of fuzzy-valued functions, Soft Comput., 24(9) (2020), 6613-6622.

[17] O. Kaleva, S. Seikkala, On fuzzy metric spaces, Fuzzy Sets and Systems, 12 (1984), 215-229.

[18] I. Kramosil, J. Michalek, Fuzzy metric and statistical metric spaces, Kybernetika, 11(5) (1975), 336-344.

[19] C. Li, Y. Zhang, J. Zhang, On statistical convergence in fuzzy metric spaces, J. Intell. Fuzzy Syst., 39(3) (2020), 3987-3993.

[20] G. D. Maio, L. Koćinac, Statistical convergence in topology, Topology Appl., 156 (2008), $28-45$.

[21] A. A. Nabiev, E. Savaş, M. Gürdal, Statistically localized sequences in metric spaces, J. Appl. Anal. Comput., 9(2) (2019), 739-746.

[22] F. Nuray, Lacunary statistical convergence of sequences of fuzzy numbers, Fuzzy Sets and Systems, 99 (1998), 353-355.

[23] F. Nuray, U. Ulusu, E. Dündar, Lacunary statistical convergence of double sequences of sets, Soft Comput., 20 (2016), 2883-2888. 
[24] S. Pehlivan, M. Gürdal, B. Fisher, Lacunary statistical cluster points of sequences, Math. Commun., 11 (2006), 39-46.

[25] A. Şahiner, M. Gürdal, F.K. Düden, Triple sequences and their statistical convergence, Selçuk J. Appl. Math., 8(2) (2007), 49-55.

[26] T. Salát, On statistically convergent sequences of real numbers, Math. Slovaca, 30(2) (1980), 139-150.

[27] E. Savaş, M. Gürdal, Certain summability methods in intuitionistic fuzzy normed spaces, J. Intell. Fuzzy Syst., 27(4) (2014), 1621-1629.

[28] E. Savaş, M. Gürdal, Generalized statistically convergent sequences of functions in fuzzy 2-normed spaces, J. Intell. Fuzzy Syst., 27 (2014), 2067-2075.

[29] E. Savaş, M. Gürdal, A generalized statistical convergence in intuitionistic fuzzy normed spaces, ScienceAsia, 41 (2015), 289-294.

[30] E. Savaş, M. Gürdal, Ideal convergent function sequences in random 2-normed spaces, Filomat, 30(3) (2016), 557-567.

[31] B. Schweizer, A. Sklar, Statistical metric spaces, Pacific J. Math., 10 (1960), 314-334.

[32] U. Yamancı, M. Gürdal, std-statistical convergence in intuitionistic fuzzy normed spaces, Notes on Intuitionistic Fuzzy Sets, 22(2) (2016), 52-58.

[33] U. Yamancı, M. Gürdal, Statistical convergence and operators on Fock space, New York J. Math., 22 (2016), 199-207.

[34] L.A. Zadeh, Fuzzy sets. Inform Control, 8 (1965), 338-353.

Suleyman Demirel University, Department of Mathematics, 32260, Isparta, Turkey

Email address: gurdalmehmet@sdu.edu.tr

Uşak University, 64100, Uşak, TURKey

Email address: ekremsavas@yahoo.com 